\title{
Coordinated Electric Vehicle Charging Based on Real-Time Pricing
}

\author{
Xinshu Wan ${ }^{1, *}$, Wangcheng $\mathrm{Zhu}^{1}$, Ning $\mathrm{Su}^{2}$ and Jiahong $\mathrm{Wu}^{3}$ \\ ${ }^{1}$ Electric Power Research Institute of Hainan Power Grid Co., Ltd., Haikou 570125, China \\ ${ }^{2}$ Qionghai Power Supply Company of Hainan Power Grid Co., Ltd., Qionghai 571400, China \\ ${ }^{3}$ Nanjing Sifang E-power Automation Co., Ltd., Nanjing 211111, China \\ ${ }^{*}$ Corresponding author
}

\begin{abstract}
Based on the existing time-of-use electricity price pricing scheme of Beijing, a real-time pricing scheme for electric vehicle charging is proposed in this paper to perform load shifting by adjusting the hourly-changed charging price. A twostage model of determining electric vehicle charging price is proposed. In the first stage, the charging load of the next day is predicted according to statistical probabilities. Together with the prediction of the base load pattern, the charging price of each hour is then optimized accordingly as a reference for the next day. In the second stage, the exact charging price is set ahead of each hour based on the power demand in previous hours and the desired load curve. The model is first built and then solved using the CPLEX software and MATLAB programming. The simulation result shows that the peak load as well as the peakvalley load difference is reduced. The charging cost of customers is also expected to be diminished.
\end{abstract}

Keywords-electric vehicle; real-time pricing; price prediction; charging schedule

\section{INTRODUCTION}

Climate change has raised worldwide concern over the greenhouse gas emitted by internal combustion engines. Electric vehicles can help improve air quality and reduce pollution. Up till now, many countries have announced regulations to promote electric vehicles and restrict the use of traditional vehicles. For example, France and Britain will ban the sale of cars burning gasoline from 2040. Car manufacturers like Volvo have announced plans to produce pure electric vehicles or vehicles using clean energy in the future. It is evident that electric vehicles will be purchased and used by more and more household in the future.

However, as pointed out by many literatures, uncoordinated charging of electric vehicles will bring serious problems to the power grid [1-3]. For instance, the charging demand of electric vehicles will coincide with the existing peak of load curve, creating a higher power consumption. The problem has two aspects. Firstly, the peak of base load and the popular hours of uncoordinated charging load appear at nearly the same time. Secondly, under the uncoordinated charging scheme, electric vehicle users tend to charge their cars simultaneously at certain hours after they have parked their vehicles. In order to alleviate the problem, the charging of electric vehicles should be coordinated.
Under the uncoordinated charging scenario, the increase of charging load will bring more uncertainties to the operation of power grid. In order to shift the charging load, two methods can be adopted: direct load control or price signal. According to economic principles, introducing lower charging prices in the valley period can encourage more power consumption and reduce peak-valley difference. Commonly used pricing schemes include time-of-use (TOU) tariff, real-time pricing (RTP) and critical period pricing (CPP). Among them, RTP is considered to be the most effective scheme [4].

The objective functions selected by existing literatures include minimization of peak-load difference [5-6], minimization of charging cost [4], maximization of power grid companies, maximization of users' satisfaction, etc. Some authors combine several of the above-mentioned objectives to create a multi-objective optimization model.

RTP is a typical method of demand side response. Many scholars have researched on this topic and have come up with several pricing models. The authors in [7] propose a RTP scheme using the power output of PV sources as input, together with the users' response to changes in price, leading the users to transfer their power demand and use the consume the output of photovoltaic energy as much as possible. In [8], the authors study the consumers' price elasticity under the RTP scheme, including self- and cross-elasticity, to realize the users' reaction to varying prices. Reference [9] come up with an iterable optimization model of determining RTP. By using the least square method, the relationship between users' demand and price of electricity is fitted. According to the price elasticity of demand, the load response model is constructed and solved to maximize the users' interest. A robust optimization model aiming at decreasing the total operational cost of charging service supplier is set up in [10]. Since the uncertainty of electricity price is considered, as long as the predicted price deviates from the prediction within a certain extent, the charging schedule can still be optimized.

The method raised in this paper differs from [11] in that the fixed reference charging curve is updated every hour, so that a more precise target curve is generated. A rolling optimization model over the next 24 hours is developed in [12] by calculating the best charging scheme every 30 minutes based on the latest charging load information. Based on this method, this paper takes the latest prediction of load into consideration. 
Moreover, the minimization of peak-valley is selected as the objective, along with the reduction of charging cost.

The coordinated charging strategy raised in this paper features: (1) a two-stage charging scheme which optimizes the charging behavior with the next 24 hours, aiming at minimizing the peak-load difference of the daily load curve; (2) generation of the electricity price of charging and prediction of EV charging load of the next day in the first stage, considering the users' demand and safety of the grid; (3) determination of the final charging price based on the latest prediction of charging load and the deviations generated in previous time slots.

The rest of the paper is formulated as follows: the two-stage RTP scheme is constructed in Section II; Section III illustrates the simulation results; the analysis and conclusion are presented in Sections IV and V respectively.

\section{MODEl CONSTRUCTION}

\section{A. Stage One}

Although many load prediction methods have been developed, still the real load will deviate from the predicted value. The factors affecting electric vehicle drivers' behavior include bad weather condition, traffic control, etc. Since the daily travelling mileage is not affected by the start time of charging, the charging demand is the same before and after optimization. Meanwhile, the price of electricity within a time slot should exceed the cost of energy generation and transmission. In order to protect the interest of electric vehicle drivers, an upper boundary should be set.

Because the charging process can only start before the end of travelling, the optimized start time of charging can only be postponed. In order to alleviate the impact on vehicle users, the delay should be made as short as possible.

Price elasticity is effective in terms of analyzing customers' response to changes in price [6]. The demand of electricity changes with the variation of price, as described by

$$
\varepsilon=\Delta D / \Delta \pi \cdot \pi_{0} / D_{0}
$$

where $\Delta D$ and $\Delta \pi$ represent the change in load and price, $\pi_{0}$ and $D_{0}$ are the initial values of load and price, and $\varepsilon$ is the price elasticity matrix. The price elasticity matrix is consisted of two parts: self-elasticity and cross-elasticity. The self-elasticity is situated at the diagonal of the matrix, while off-diagonal elements are cross-elasticities. Because the daily charging demand must be satisfied, the shift of charging load caused by self-elasticity is not considered. The change of load at time slot $t_{i}$ caused by change of price at time slot $t_{j}$ is denoted by $\varepsilon_{i j}$. This paper adopts the electricity price elasticity proposed by [13]. As the RTP changes every hour, the size of the price elasticity matrix is $24 \times 24$. The total change of load in hour $i$ caused by changes of price in all the other hours is

$$
\Delta D\left(t_{i}\right)=\sum_{j=1}^{24} \varepsilon_{i j} \Delta \pi_{j} D_{0}\left(t_{i}\right) / \pi_{0}
$$

where $\Delta \pi_{j}=\pi_{j}-\pi_{j 0} ; \pi_{j}$ and $\pi_{j 0}$ represent the proposed real-time price and the original TOU price respectively. The changed demand of charging power at hour $i$ becomes

$$
D\left(t_{i}\right)=D_{0}\left(t_{i}\right)+\Delta D\left(t_{i}\right)
$$

The optimization model is constructed as follows:

$$
\operatorname{minimize} \quad\left[\max D_{T}\left(t_{i}\right)-\min D_{T}\left(t_{i}\right)\right]
$$

$$
\text { subject to } \quad \sum_{i=1}^{24} D\left(t_{i}\right)=\sum_{i=1}^{24} D_{0}\left(t_{i}\right)
$$

$$
D\left(t_{i}\right) \leq P_{\max }\left(t_{i}\right)
$$

$$
\begin{gathered}
\sum_{i=1}^{24} \pi\left(t_{i}\right)=\sum_{i=1}^{24} \pi_{0}\left(t_{i}\right) \\
\sum_{i=1}^{24} D\left(t_{i}\right) \times \pi\left(t_{i}\right) \leq \sum_{i=1}^{24} D_{0}\left(t_{i}\right) \times \pi_{0}\left(t_{i}\right)
\end{gathered}
$$

Equation (5) means that the total charging power demand is not changed before and after optimization. $P_{\max }\left(t_{i}\right)$ in (6) denotes the maximum charging power restricted by the ratings of lines and transformers. Eq.(7) represents the mean price of electricity before and after the optimization is constant. (8) means that the total cost after the optimization should be lower than before.

\section{B. Stage Two}

By solving the optimization model in stage one, the predicted charging power is obtained and used as the reference of the second stage. The objective of the second stage is to make the actual charging curve as close to the day-ahead predicted value as possible. The reference curve generated in stage one is idealized in that charging load in several hours are set to be zero. In reality, drivers will charge their vehicles in day time due to urgent charging need. The model assumes that the total charging power within a day in the studied region is nearly constant, which is evident that drivers tend to charge their batteries full at the end of the day. Therefore, the total charging demand of the remaining hours of a day can be predicted by the existing charging data of each of the past time slots. When the latest prediction deviates from the day-ahead reference value, actions should be taken to reduce the difference. The self-elasticity of electricity is utilized to alter the electricity price and charge the charging demand.

The reference is updated every hour to maintain its precision. The time step of one hour is selected to accommodate the hourly changed RTP. When updating the prediction, the actual charging load and other load are added into the model to generate a new reference curve. When the 
above-mentioned data are unavailable, the previous obtained prediction is used.

The revision of the reference load curve is as follows:

Firstly, the accumulated deviance of the actual curve and the day-ahead predicted values are calculated and evenly distributed to all the remaining hours of that day. Namely, for the accumulated charging demand not served, certain amount of predicted will be added to the prediction curve to be the new reference curve of future hours. As for the exceedingly used charging demand, some load will be diminished from the old curve. Since even distributions is adopted, even the latest data cannot be obtained, the daily charging reference can still be kept until the end of the day.

Secondly, the base load curve may deviate from predicted. However, methods with high precision has been developed and can be used to accurately predict the total load of the next few hours. The prediction method of the base load curve exceeds the scope of this paper. It is assumed that the base load demand of the next few hours can be predicted with high accuracy. This paper focuses on the adjustment of reference charging curve according to changes of base load.

The reference charging load is updated using the following equation:

$$
D_{p}(h)=D_{p 0}(h)+\left[\sum_{i=1}^{h-1} D_{p 0}\left(t_{i}\right)-\sum_{i=1}^{h-1} D_{a}\left(t_{i}\right)\right] /(25-h),(9)
$$

where $D_{p 0}\left(t_{i}\right)$ denotes the original reference charging load for the $i^{\text {th }}$ hour, and $D_{p 0}\left(t_{i}\right)$ is the actual charging power of the $i^{\text {th }}$ hour.

In order to calculate the change of charging price, the shifted value of charging load should be obtained. The available charging power that can be supplied of each hour consists of two parts. One is brought by the deviation of predicted and actual values of the base load. The other is determined by the accumulated deviation of the actual and predicted charging load. The charging load of each hour is obtained by

$$
\Delta D(h)=\Delta D_{p}(h)+\Delta L_{p}(h) .
$$

Considering the reduction of load during peak hours, when the actual charging power is less than predicted, the abundant power will not be added to the available charging power. According to the load consumption pattern, 17.00 to 21.00 are selected as peak hours. The effect on the available charging power is given by

$$
\Delta D(h)=\left\{\begin{array}{ll}
D_{p}(h)-D_{p 0}(h)+L_{p}(h)-L_{p 0}(h) & h \notin[17,21] \\
D_{p}(h)-D_{p 0}(h) & h \in[17,21]
\end{array},\right.
$$

where $L_{p}\left(t_{i}\right)$ denotes the latest prediction of base load in the $i^{\text {th }}$ hour, and $L_{p 0}\left(t_{i}\right)$ is the original prediction of base load in the $i^{\text {th }}$ hour used in stage one.
The change of charging price can be calculated using

$$
\Delta \pi(h)=\pi_{0}(h) \cdot \Delta D(h) /\left[\varepsilon(h) \cdot D_{0}(h)\right],
$$

where $\varepsilon(h)$ represents the self-elasticity of electricity in the hour of $h$.

\section{SimUlation RESUlts}

\section{A. Uncoordinated Charging}

Because fast charging is usually used when in urgent scenarios, its demand is not affected by price. In order to study the optimal RTP, this paper focuses on the normal charging behavior of electric vehicles, which is commonly used by consumer vehicles. Since the power is relatively small, its demand is sensitive to changes in price.

According to the national household travel survey conducted by the US Department of Transportation, although the daily travel behavior of a single vehicle is hard to predict, the travelling of a flock of vehicles has some regular patterns. It is found that the ending time of the last journey of a day coincides with normal distribution [14], namely

$$
T_{\text {return }}\left(V_{i}\right) \sim N\left(\mu_{T}, \sigma_{T}^{2}\right)
$$

Meanwhile, the daily travelling mileage can be approximated by log-normal distribution, namely

$$
\operatorname{distance}\left(V_{i}\right) \sim \operatorname{lognormal}\left(\mu_{d}, \sigma_{d}^{2}\right) \text {. }
$$

Moreover, this paper further assumes that the normal charging power is evenly distributed between the lower and higher boundary

$$
D_{\text {vehicle }}\left(V_{i}\right) \sim U\left[P_{\min }, P_{\max }\right] \text {, }
$$

where $P_{\min }$ and $P_{\max }$ represent the minimum and maximum charging power.

Assume that the total number of electric vehicles in a certain region is 50,000 . The daily travelling distance distribution has a mean value of $20 \mathrm{~km}$ and a variance of $8.8 \mathrm{~km}$. The start time of charging follows the normal distribution whose mean value equals $17.6 \mathrm{~h}$ and variance equal $3.4 \mathrm{~h}$. The lower and upper boundaries of normal charging power are $2 \mathrm{~kW}$ and $5 \mathrm{~kW}$ respectively.

The typical base load data of an area is shown in Table I. The total load curve can be obtained by combining the base load and charging load. According to equations (13)-(15), the daily charging load can be obtained using Monte-Carlo Simulation method. The result is shown in Figure I. 
TABLE I. BASE LOAD OF EACH HOUR

\begin{tabular}{|c|c|c|c|}
\hline Hour & Load/MW & Hour & Load/MW \\
\hline 1 & 511 & 13 & 682.5 \\
\hline 2 & 532.5 & 14 & 692.5 \\
\hline 3 & 520 & 15 & 688 \\
\hline 4 & 491 & 16 & 686 \\
\hline 5 & 543.5 & 17 & 729 \\
\hline 6 & 566 & 18 & 735 \\
\hline 7 & 596 & 19 & 778 \\
\hline 8 & 643.5 & 20 & 775 \\
\hline 9 & 717.5 & 21 & 729 \\
\hline 10 & 734 & 22 & 681 \\
\hline 11 & 752.5 & 23 & 581.5 \\
\hline 12 & 670 & 24 & 553.5 \\
\hline
\end{tabular}

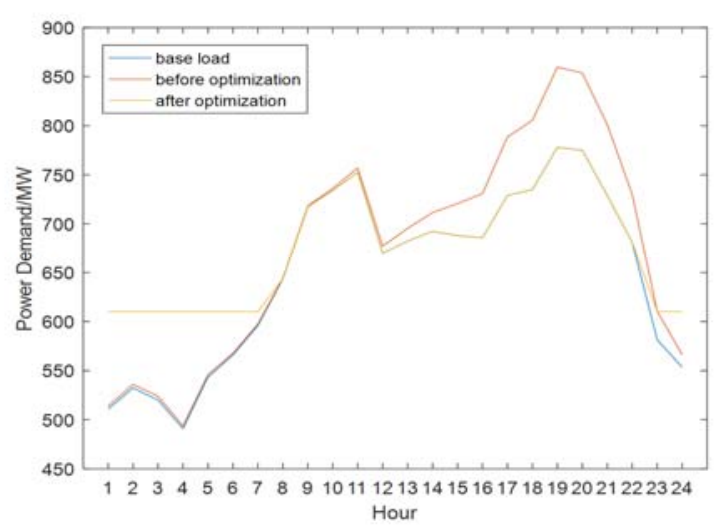

FIGURE I.

BASE LOAD AND CHARGING LOAD.

\section{B. Coordinated Charging}

After implementing the optimization model proposed in stage one, the resulting load curve can be obtained. The new total load curve is illustrated by the yellow line in Figure I. The corresponding RTP of each hour is presented in Figure II.

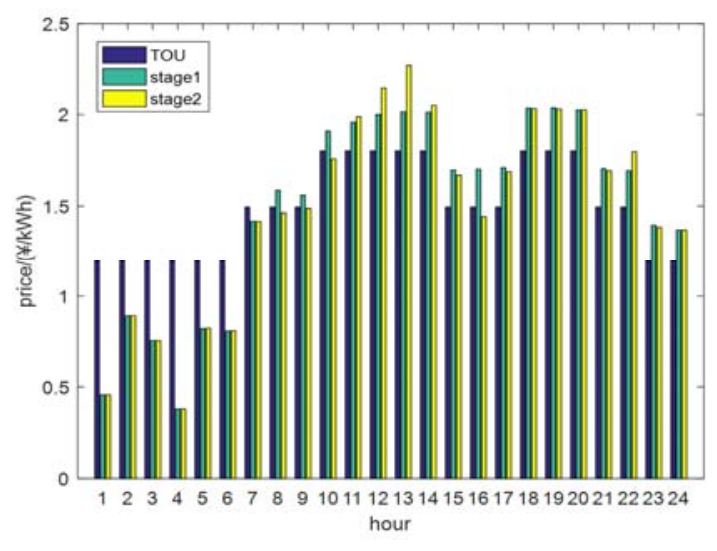

FIGURE II

PRICE OF DIFFERENT STAGES

\section{ANALYSIS}

As can be seen clearly from the red line in Figure I, because the peak charging demand of electric vehicles appears at the same time with the maximum of load demand, the peak increases from $778 \mathrm{MW}$ to $830.9 \mathrm{MW}$, rising by $6.8 \%$.

After optimization, a large amount of charging power is shifted to the time period when base load is low and electricity is cheap. The minimum total power usage rises to $610.22 \mathrm{MW}$. Meanwhile, the ideal charging demand during peak hours is decreased to zero, avoiding the increase of maximum load demand. In order to encourage drivers to perform loadshedding and valley-filling, the charging price is modified accordingly. Since the base load during evening are varied between hours, the optimized prices of electricity also differ from each other to flatten the total load curve and avoid a new peak load in the evening. As is restricted by the time order, the start time of charging can only be postponed. Consequently, the charging load are shifted to time slots when base load is low, namely midnight and early morning. The maximum decrease of charging price is $68 \%$ and the price drops to $0.3837 \mathrm{yuan} / \mathrm{kWh}$, which creates enough stimulation for customers to delay their charging behavior. In order to balance the price, the price of electricity of charging increases in day time. The maximum price appears at 19.00 when the price increases from 1.8044 yuan $/ \mathrm{kWh}$ to 2.0364 yuan $/ \mathrm{kWh}$. The peak-valley difference drops from 405.9MW to $309.0 \mathrm{MW}$, fulfilling the objective of load-flattening.

Under the uncoordinated scenario, the total cost of charging is $960,518.5$ yuan. After stage one, the cost becomes 331694.6 yuan, which results from the large amount of load shifted from peak hours to valley hours. Because of the drop of charging cost, customers of electric vehicles gain more benefit. At the same time, since the amount of energy purchased from power grid during peak hours are reduced, the cost of charging service providers also decreases. However, their profit is influenced by the price users pay for charging. Under the pricing regulations, these service providers can gently increase charging prices of several hours to keep their balance. As for grid companies, with the drop of peak load and decrease in peak-valley difference, the reliability of service is boosted, along with an increase in the utilization rate of generators. As the load changed in stage two, the economic performance cannot be evaluated. However, the second stage takes the latest load prediction into account, creating a more accurate reflection of the actual load on the charging price.

\section{CONCLUSIONS}

The results show that the proposed real-time pricing mechanism has a positive influence on electric vehicle users and power grid. Charging service providers can also keep their financial balancing by setting a higher price during peak hours. Using price signals to control the charging demand is a simple, flexible and effective method. It has a potential of implementation in the future. The two-stage pricing model takes advantage of both the day-advance and hour-advance mechanisms, announcing the relatively accurate price ahead of time and making small variations according to the precise predictions. 


\section{ACKNOWLEDGEMENT}

This research is supported by China Southern Power Grid Corporation (Research and Application of Key Technologies of Intelligent Cloud Service in Hainan Power Grid).

\section{REFERENCES}

[1] ZHANG Cong. The Related Study on Impacts And Utilization of Largescale Electric Vehicles Integration into Power Grid[D]. Wuhan: Huazhong University of Science and Technology, 2015.

[2] HU Zechun, SONG Yonghua, XU Zhiwei, et al. Impacts and Utilization of Electric Vehicles Integration Into Power Systems[J]. Proceedings of the CSEE, 2012, 32(04):1-10.

[3] GAO Ciwei, ZHANG Liang. A Survey of Influence of Electrics Vehicle Charging on Power Grid[J]. Power System Technology, 2011, 35(02):127-131.

[4] Vagropoulos S, Balaskas G, Bakirtzis A. An investigation of plug-in electric vehicle charging impact on power systems scheduling and energy costs[J]. IEEE Transactions on Power Systems, 2017, 32(3):1902-1912.

[5] Singh M, Thirugnanam K, Kumar P, et al. Real-Time Coordination of Electric Vehicles to Support the Grid at the Distribution Substation Level[J]. IEEE Systems Journal. 2015, 9(3):1000-1010.

[6] Kim S J, Giannakis G B. Real-time electricity pricing for demand response using online convex optimization[C]// Innovative Smart Grid Technologies Conference. IEEE, 2014:1-5.

[7] SHI Leilei, HU Jianzhao, HAN Shengfeng. An electricity price excited load transfer strategy for improving PV penetration ability to power systems[J]. Power System Protenction and Control, 2016, 44(23): 153157.

[8] HUANG Haixin, DENG Li, WEN Feng. Customer Response Behavior Based on Real-Time Pricing[J]. Electric Power Construction, 2016, 37(2):63-68.

[9] MENG Xiang, MA Xiaojun, YU Chao, et al. Real-Time Electricity Price Optimizaiton Model Based on User Response[J]. Electrician Abstracts, 2014(03):58-60.

[10] Soroudi A, Keane A. Robust optimization based EV charging[C]// Electric Vehicle Conference. IEEE, 2015:1-6.

[11] Sundstrom O, Binding C. Flexible Charging Optimization for Electric Vehicles Considering Distribution Grid Constraints[J]. IEEE Transactions on Smart Grid. 2012, 3(1):26-37.

[12] O'Connell A, Flynn D, Keane A. Rolling Multi-Period Optimization to Control Electric Vehicle Charging in Distribution Networks[J]. IEEE Transactions on Power Systems. 2014, 29(1): 340-348.

[13] Taylor T N, Schwarz P M, Cochell J E. 24/7 Hourly Response to Electricity Real-Time Pricing with up to Eight Summers of Experience[J]. Journal of Regulatory Economics. 2005, 27(3): 235-262.

[14] Zhang W, Zhang D, Mu B, et al. Decentralized Electric Vehicle Charging Strategies for Reduced Load Variation and Guaranteed Charge Completion in Regional Distribution Grids[J]. Energies. 2017, 10(2): 147. 